\title{
Preface: ICPPP-17
}

\section{Chinhua Wang ${ }^{1}$ • Bincheng $\mathbf{L i}^{2}$. Shuyi Zhang ${ }^{3}$}

The Seventeenth International Conference on Photothermal and Photoacoustic Phenomena (17th ICPPP) was held in Suzhou, Jiangsu, China from October 20 to 24, 2013. This biennial ICPPP, started in the beginning of the 1980 s, is widely recognized as the major venue for the dissemination of recent and significant research results and exchange of creative ideas in these traditional areas, as well as in new and exciting outgrowths of this interdisciplinary field. The 17th ICPPP brought together scientists, engineers, and developers from a very broad spectrum of disciplines whose common area of interests and activities is related with the development and use of methodologies involving the generation, propagation, and detection of acoustic, thermal, and general diffusion waves, with applications in materials science, instrumentation, technology, biomedicine, and biology.

This event was organized by the Institute of Modern Optical Technologies, Soochow University, and the Key Lab of Beam Manipulation, Institute of Optics and Electronics of the Chinese Academy of Science. During the conference, 212 papers from more than 20 countries were presented, 9 corresponding to plenary papers, 2 to tutorial papers, 21 to invited papers, 78 to oral contributions, and 102 to poster contributions. All these papers are distributed in different directions of the photoacoustic and photothermal field, including (1) scanning photoacoustic and photothermal imaging, tomography, and depth profiling; (2) photoacoustic and photothermal applications in biology, agriculture, biomedicine, and environmental sensing; (3) electronic, optical materials, semiconductors, thin films, and devices; (4) new instrumentation and

Chinhua Wang

chinhua.wang@suda.edu.cn

1 Institute of Modern Optical Technologies, Soochow University, Suzhou 215006, China

2 Key Laboratory of Beam Manipulation, Chinese Academy of Science, Chengdu 610209, China

3 Institute of Acoustics, Nanjing University, Nanjing 210093, China 
methodology and new applications based on photoacoustic and photothermal phenomena; (5) nondestructive testing, thermophysical properties, and industrial applications; (6) dynamic ultrafast phenomena; and (7) nanostructures and nanomaterials.

The International Photoacoustic and Photothermal Association (IPPA) Prizes, a major honor within the photothermal science community for outstanding achievements in this field of research and development, were awarded at the 17th ICPPP. The Senior Prize was presented to Professor Josef Pelzl (Ruhr-University, Germany), "for pioneering research on phase-resolved photoacoustic spectroscopy, photothermally modulated magnetic resonance, thermal detection of ferromagnetic resonance, ..., and contributions to the theory of both photoacoustic and photothermal science; for mentoring of young scientists, and initiation of cooperative research projects that have resulted in dissemination of photoacoustic and photothermal methods worldwide." The James Smith IPPA Prize was awarded to Professor Peter Hess (University of Heidelberg, Germany) "for advances in the photoacoustic trace detection of gases; for pioneering work in laser generation of surface acoustic waves (2D) and wedge waves (1D), ..., the discovery of solitary surface and supersonic leaky wedge waves, and the exploration of these waves for the determination of mechanical properties, nondestructive evaluation, and the physics of impulsive fracture." The Junior IPPA Prize was awarded to Dr. Edilberto Jose Ordoñez Miranda (Ecole Centrale Paris, France) "for his outstanding contributions to the understanding and modeling of heat transport at the micro and nanoscales... for finding a novel model of heat conduction based on the Boltzmann transport equation... for his pioneering studies on the contribution of surface electromagnetic waves to the thermal conductivity of materials..."

First and foremost, we want to thank all the participants of the 17th ICPPP, for contributing to a very successful, informative, and challenging program. In particular, it is a pleasure to acknowledge the expertise and dedication of the Scientific Advisory Committee and the many individuals who contributed to the successful organization of the Symposium and to the preparation of this special issue. Among these are the plenary, tutorial, invited speakers and chairs of the sessions: Andreas Mandelis (University of Toronto, Canada), Lihong V. Wang (Washington University, USA), Oliver Wright (Hokkaido University, Japan), Frank K. Tittel (Rice University, USA), Xinwei Wang (Iowa State University, USA), Vitalyi Gusev (Université du Maine, France), Peter Hess (University of Heidelberg, Germany), Daniele Fournier (CNRS, France), Vasilis Ntziachristos (Technische Universität München, Germany), Arantza Mendioroz (Universidad del País Vasco UPV/EHU, Spain), Frederic Decremps (Université Pierre et Marie Curie, France), Peter Burgholzer (Doppler Laboratory for Photoacoustic Imaging and Laser Ultrasonics, Austria), Jun Shen (National Research Council Canada, Canada), Che-hua Yang (National Taipei University of Technology, Taiwan), Roberto Li Voti (Sapienza Università di Roma, Italy), Andrey V. Akimov (University of Nottingham, UK), P-Olivier Chapuis (CNRS, France), Juejun Hu (University of Delaware, USA), Juan Jose Alvarado-Gil (CINVESTAV Unidad Mérida, México), Heng Ban (Utah State University, USA), A. K. Chaudhary (University of Hyderabad, India), Mikhail Proskurnin (Lomonosov Moscow State University, Russia), Christ Glorieux (KU Leuven, Belgium), Bertrand Audoin (Université de Bordeaux, France), Mladen Franko (University of Nova Gorica, Slovenia), Ugo Zammit (Università di Roma, ITALY), Bruno Palpant (Ecole Centrale Paris, France), Shuyi Zhang (Nanjing Univer- 
sity, China), Zhonghua Shen (Nanjing University of Science and Technology, China), Jan Thoen (KU Leuven, Belgium), Bincheng Li (Chinese Academy of Science, China), Josef Pelzl (Ruhr-University, Germany), Yuetao Yang (Nanjing University, China), and Boris Majaron (Josef Stefan Institute, Slovenia).

Special thanks to the members of the staff, Hong Qu, Yuan Zhang, Feng Xu, Maocheng Wu, Bing Cao, Jing Cao, Zhonghui Chen, Rui Tai, Miao Wang, Jingpei $\mathrm{Hu}$, and Xiaonan Zhao for their strong support for assisting in the various tasks before, during, and after the conference. We also would like to express our special gratitude to individuals whose consultation, advice, and commitment with the photothermal and photoacoustic community were crucial to make the conference a success, Andreas Mandelis, Juan José Alvarado-Gil, and Christ Glorieux.

All papers published in this special issue have undergone a standard review process of the International Journal of Thermophysics. We would like to express thanks and appreciation to all the members of the board of Invited Guest Editors and reviewers that carried out highly valuable work that guaranteed the high scientific level of the proceedings. We are especially grateful to Dr. William M. Haynes, Editor-in-Chief of the International Journal of Thermophysics, for his outstanding contribution to publishing this issue, whose kindness and assistance are difficult to be overestimated. Thanks to him and the positive role that Prof. Andreas Mandelis played as Senior Editor of the Journal during the conference preparation, the possibility of publishing the proceedings of the 17th ICPPP as a special issue of the International Journal of Thermophysics has become a reality. We are sure that the great variety of topics addressed in the proceedings will be useful not only for researchers interested in the photothermal science area but also for a wider audience.

We gratefully acknowledge the sponsorship of the Natural Science Foundation of China, the project of the Priority Academic Program Development (PAPD) of Jiangsu Higher Education Institutions, Office of Human Resources Development of Suzhou Municipality, Suzhou Association for Science and Technology, Soochow University, the Key Laboratory of Beam Manipulation of Chinese Academy of Science, Jiangsu Optical Society, the Acoustical Society of Jiangsu Province, and Laser \& Optical Engineering Society of Suzhou.

Finally, our best wishes for the organizers of the next 18th ICPPP in 2015 in Novi Sad Serbia. 